\title{
Mercury Monohalides: Suitability for Electron Electric Dipole Moment Searches
}

\author{
V.S. Prasannaa ${ }^{1}$, A.C. Vutha ${ }^{2}$, M. Abe Ab, $^{3,4}$ and B.P. Das ${ }^{1}$ \\ ${ }^{1}$ Indian Institute of Astrophysics, Koramangala II block, Bangalore-560034, India \\ ${ }^{2}$ Dept. of Physics and Astronomy, York University, Toronto ON M3J 1P3, Canada \\ ${ }^{3}$ Tokyo Metropolitan University, 1-1, Minami-Osawa, Hachioji-city, Tokyo 192-0397, Japan and \\ ${ }^{4}$ JST, CREST, 4-1-8 Honcho, Kawaguchi, Saitama 332-0012, Japan
}

\begin{abstract}
Heavy polar diatomic molecules are the primary tools for searching for the $T$-violating permanent electric dipole moment of the electron (eEDM). Valence electrons in some molecules experience extremely large effective electric fields due to relativistic interactions. These large effective electric fields are crucial to the success of polar-molecule-based eEDM search experiments. Here we report on the results of relativistic $a b$ initio calculations of the effective electric fields in a series of molecules that are highly sensitive to an eEDM, the mercury monohalides ( $\mathrm{HgF}, \mathrm{HgCl}, \mathrm{HgBr}$, and $\mathrm{HgI})$. We study the influence of the halide anions on $\mathcal{E}_{\text {eff }}$, and identify $\mathrm{HgBr}$ and $\mathrm{HgI}$ as interesting candidates for future electric dipole moment search experiments.
\end{abstract}

Violation of time-reversal $(T)$ symmetry is an essential ingredient to explain the matter-antimatter asymmetry of the universe [1, 2]. As Standard Model sources of $T$-violation are inadequate to explain the observed asymmetry, it is imperative to look beyond it. The strongest limits on $T$-violation arising from new particles and interactions outside the Standard Model are set by searches for the permanent electric dipole moments of fundamental particles [3, 4, like that of the electron $\left(d_{e}\right)$. A strong constraint on the electron's electric dipole moment (eEDM), $d_{e}<10^{-28} e \mathrm{~cm}$, has been set by the experiment with ThO molecules [5], and improvements of a few orders of magnitude are forecast in the near future [5, 6]. The eEDM experiments take advantage of the large effective electric field (often $\gtrsim 10^{10} \mathrm{~V} / \mathrm{cm}$ ) experienced by an electron in a polarized heavy polar molecule, which leads to a measurable energy shift, $\Delta E \propto d_{e} \mathcal{E}_{\text {eff }}$. The effective electric field, $\mathcal{E}_{\text {eff }}$, arises from the relativistic interactions of the eEDM with the electric fields of all the other charged particles in the molecule. This effect, whereby molecules polarized by $\sim \mathrm{kV} / \mathrm{cm}$ laboratory fields cause $>10 \mathrm{GV} / \mathrm{cm}$ to be applied to a valence electron, is the reason for the high precision achievable in molecule-based eEDM experiments.

The value of $\mathcal{E}_{\text {eff }}$ for a molecule has to be obtained from relativistic many-body calculations in order to convert experimentally measured frequency shifts into eEDM values. A common heuristic that is used to estimate $\mathcal{E}_{\text {eff }}$ in molecules, motivated from eEDM enhancement scaling in atoms, is that $\mathcal{E}_{\text {eff }} \propto Z_{+}^{3}$, where $Z_{+}$is the charge of the (usually heavier) cationic atom's nucleus. But molecules are not atoms. This heuristic ignores the anions which can play an important role. An improved understanding of the mechanisms leading to $\mathcal{E}_{\text {eff }}$ in relativistic polar molecules will lead to better choices of candidate molecules for future eEDM experiments.

In this work, we focus on the $\mathcal{E}_{\text {eff }}$ for a class of heavy polar molecules, the mercury monohalides, in order to test their suitability for eEDM searches. The properties of these systems can be evaluated fairly accurately, as they have a single valence electron. The fact that they are sensitive to eEDMs in their ground electronic states (unlike molecules with metastable eEDM-sensitive states which require more complicated descriptions [7]), makes them suitable test cases for high-precision calculations. $\mathrm{HgF}$ has one of the largest reported $\mathcal{E}_{\text {eff }}[8$, making this series of $\mathrm{HgX}$ molecules particularly interesting as potential candidates for future eEDM experiments. The heavier $\mathrm{Hg}$ monohalides $(\mathrm{HgCl}, \mathrm{HgBr}, \mathrm{HgI})$ are more electrically polarizable than $\mathrm{HgF}$, which translates to a more effective use of $\mathcal{E}_{\text {eff }}$ and better control over systematic effects. This, in addition to better prospects for their production and efficient detection, makes the investigation of their $\mathcal{E}_{\text {eff }}$ values very promising for future eEDM experiments.

The expression for $\mathcal{E}_{\text {eff }}$ in terms of an effective eEDM operator, $H_{\mathrm{EDM}}^{\mathrm{eff}}$ is given by 9

$$
\begin{aligned}
\mathcal{E}_{\text {eff }} & =\frac{2 i c}{e} \sum_{j=1}^{N_{e}}\left\langle\psi\left|\beta \gamma_{5} p_{j}^{2}\right| \psi\right\rangle \\
& =\frac{1}{d_{e}}\left\langle\psi\left|H_{\mathrm{EDM}}^{\mathrm{eff}}\right| \psi\right\rangle
\end{aligned}
$$

Here, $\mathrm{c}$ is the speed of light, e is the charge of the electron, $N_{e}$ refers to the number of electrons in the molecule, $\beta$ is one of the Dirac matrices, $\gamma_{5}$ is the product of the Dirac matrices, and $\mathbf{p}_{j}$ is the momentum of the $j^{\text {th }}$ electron. $\psi$ is the wavefunction of a molecular state. The above expression casts the eEDM Hamiltonian in terms of one-electron operators, which makes it convenient for computations. Further details of the derivation of this form can be found in 9 .

To obtain the molecular wavefunction $|\psi\rangle$, we use a relativistic coupled cluster (RCC) method [10, 11]. The coupled cluster wavefunction can be written as

$$
|\psi\rangle=e^{T}\left|\Phi_{0}\right\rangle
$$

Here, $\left|\Phi_{0}\right\rangle$ refers to the Dirac-Fock (DF) wavefunction, which is built from single particle four-component spinors. This is the model wavefunction for the coupled cluster calculations, and is taken to be a single determinant 
corresponding to an open shell doublet. $T$ is the cluster operator. In this work, we use the CCSD (Coupled Cluster Singles and Doubles) approximation, where $T=T_{1}+$ $T_{2}$, where $T_{1}$ and $T_{2}$ are the single (S) and double (D) excitation operators respectively. They are given by

$$
\begin{aligned}
& T_{1}=\sum_{i, a} t_{i}^{a} a^{\dagger} i \\
& T_{2}=\sum_{a>b, i>j} t_{i j}^{a b} a^{\dagger} b^{\dagger} j i .
\end{aligned}
$$

Here, $t_{i}^{a}$ and $t_{i j}^{a b}$ are called the cluster amplitudes. In our notation, $i, j, k, \ldots$ refer to holes and $a, b, c, \ldots$ refer to particles. When $a^{\dagger} i$ acts on a state, a hole $i$ is destroyed from that state, and a particle $a$ is created. The action of $a^{\dagger} i$ on a model state, $\left|\Phi_{0}\right\rangle$ results in a state denoted by $\left|\Phi_{i}^{a}\right\rangle$.

The CCSD amplitude equations are

$$
\begin{aligned}
\left\langle\Phi_{i}^{a}\left|e^{-T} H_{N} e^{T}\right| \Phi_{0}\right\rangle & =0 \\
\left\langle\Phi_{i j}^{a b}\left|e^{-T} H_{N} e^{T}\right| \Phi_{0}\right\rangle & =0
\end{aligned}
$$

The term $e^{-T} H_{N} e^{T}$ can be written as $\left\{H_{N} e^{T}\right\}_{c}$, due to the linked cluster theorem [12, 13. $H_{N}$ is the normalordered Hamiltonian [14. The subscript $c$ means that each term in the expression is connected. The effective fields are calculated by using only the linear terms in the coupled cluster wavefunction, since the dominant contributions come from them. Hence we evaluate

$$
\begin{aligned}
\mathcal{E}_{\mathrm{eff}} & =\left\langle\Phi_{0}\left|\left(1+T_{1}+T_{2}\right)^{\dagger}\left(\frac{H_{\mathrm{EDM}}^{\mathrm{eff}}}{d_{e}}\right)_{N}\left(1+T_{1}+T_{2}\right)\right| \Phi_{0}\right\rangle_{c} \\
& +\left\langle\Phi_{0}\left|\frac{H_{\mathrm{EDM}}^{\mathrm{eff}}}{d_{e}}\right| \Phi_{0}\right\rangle
\end{aligned}
$$

We note that although the expectation value uses the linearized expansion of the coupled cluster wavefunction, the amplitudes are evaluated at the CCSD level.

We performed our calculations by combining and modifying the UTCHEM [15,17] and the DIRAC08 [18] codes. We used the $C_{8}$ point group, which reduces the computational time for the atomic-to-molecular orbital integral transformations. A summary of our calculations, both at the DF and the CCSD level, are given in Table I, and the results plotted in Figure 1. We find that the values for $\mathcal{E}_{\text {eff }}$ are very large for all of the chosen mercury halides, and are typically about one and a half times that of ThO [7] and about five times that of $\mathrm{YbF}[9]$. This can be attributed to the fact that there is strong mixing between the valence 6 s and the virtual $6 \mathrm{p}$ orbital.

We chose uncontracted correlation-consistent, polarized

\begin{tabular}{|c|c|c|c|c|}
\hline Molecule & Method & Basis & $T_{1, \text { dia }}$ & $\mathcal{E}_{\text {eff }}(\mathrm{GV} / \mathrm{cm})$ \\
\hline $\mathrm{HgF}$ & DF & $\begin{array}{c}\mathrm{Hg}: 22 \mathrm{~s}, 19 \mathrm{p}, 12 \mathrm{~d}, 9 \mathrm{f}, 1 \mathrm{~g} \\
\mathrm{~F}: 9 \mathrm{~s}, 4 \mathrm{p}, 1 \mathrm{~d}\end{array}$ & - & 104.25 \\
\hline $\mathrm{HgCl}$ & DF & $\begin{array}{c}\mathrm{Hg}: 22 \mathrm{~s}, 19 \mathrm{p}, 12 \mathrm{~d}, 9 \mathrm{f}, 1 \mathrm{~g} \\
\mathrm{Cl}: 12 \mathrm{~s}, 8 \mathrm{p}, 1 \mathrm{~d}\end{array}$ & - & 103.57 \\
\hline $\mathrm{HgBr}$ & DF & $\begin{array}{c}\mathrm{Hg}: 22 \mathrm{~s}, 19 \mathrm{p}, 12 \mathrm{~d}, 9 \mathrm{f}, 1 \mathrm{~g} \\
\mathrm{Br}: 14 \mathrm{~s}, 11 \mathrm{p}, 6 \mathrm{~d}\end{array}$ & - & 97.89 \\
\hline $\mathrm{HgI}$ & DF & $\begin{array}{c}\mathrm{Hg}: 22 \mathrm{~s}, 19 \mathrm{p}, 12 \mathrm{~d}, 9 \mathrm{f}, 1 \mathrm{~g} \\
\mathrm{I}: 8 \mathrm{~s}, 6 \mathrm{p}, 6 \mathrm{~d}\end{array}$ & - & 96.85 \\
\hline $\mathrm{HgF}$ & CCSD & $\begin{array}{c}\mathrm{Hg}: 22 \mathrm{~s}, 19 \mathrm{p}, 12 \mathrm{~d}, 9 \mathrm{f}, 1 \mathrm{~g} \\
\mathrm{~F}: 9 \mathrm{~s}, 4 \mathrm{p}, 1 \mathrm{~d}\end{array}$ & 0.0268 & 115.42 \\
\hline $\mathrm{HgCl}$ & CCSD & $\begin{array}{c}\mathrm{Hg}: 22 \mathrm{~s}, 19 \mathrm{p}, 12 \mathrm{~d}, 9 \mathrm{f}, 1 \mathrm{~g} \\
\mathrm{Cl}: 12 \mathrm{~s}, 8 \mathrm{p}, 1 \mathrm{~d}\end{array}$ & 0.0239 & 113.56 \\
\hline $\mathrm{HgBr}$ & CCSD & $\begin{array}{c}\mathrm{Hg}: 22 \mathrm{~s}, 19 \mathrm{p}, 12 \mathrm{~d}, 9 \mathrm{f}, 1 \mathrm{~g} \\
\mathrm{Br}: 14 \mathrm{~s}, 11 \mathrm{p}, 6 \mathrm{~d}\end{array}$ & 0.0255 & 109.29 \\
\hline $\mathrm{HgI}$ & CCSD & $\begin{array}{c}\mathrm{Hg}: 22 \mathrm{~s}, 19 \mathrm{p}, 12 \mathrm{~d}, 9 \mathrm{f}, 1 \mathrm{~g} \\
\mathrm{I}: 8 \mathrm{~s}, 6 \mathrm{p}, 6 \mathrm{~d}\end{array}$ & 0.0206 & 109.30 \\
\hline
\end{tabular}
valence double zeta (ccpvdz) basis sets for $\mathrm{F}, \mathrm{Cl}$ and $\mathrm{Br}$ [19], and Dyall's c2v basis sets for $\mathrm{Hg}$ [20]. We use Dyall's basis for I [20]. We use Gaussian Type Orbitals (GTO), which are kinetically balanced [21]. Our calculations were performed without freezing any of the core orbitals. We
TABLE I. Summary of the calculated results $\left(\mathcal{E}_{\text {eff }}\right)$ of the present work.

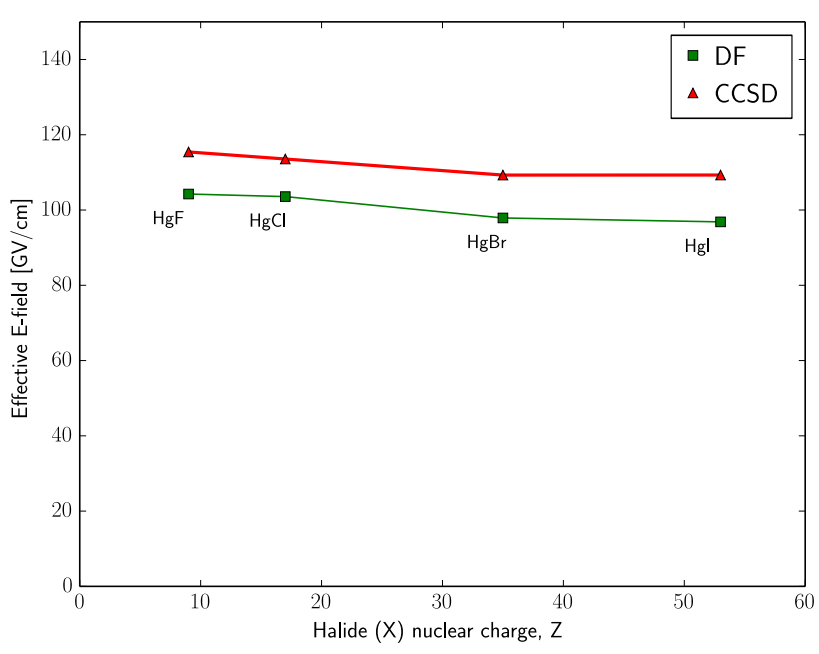

FIG. 1. Effective electric fields, $\mathcal{E}_{\text {eff }}$, for $\mathrm{HgX}$ molcules calculated using Dirac-Fock wavefunctions (DF, green squares), and using coupled cluster with singles and doubles (CCSD, red triangles). Solid lines are guides to the eye. The difference between DF and CCSD values indicates the contribution of electron correlation to $\mathcal{E}_{\text {eff }}$.

used the following bond lengths (in $\mathrm{nm}$ ) for our calculations: $\mathrm{HgF}(0.200686)$ [22], $\mathrm{HgCl}(0.242), \mathrm{HgBr}$ (0.262), $\mathrm{HgI}(0.281)$ [23].

We have also reported the $T_{1}$ diagnostics (denoted as $\left.T_{1, \text { dia }}\right)$, a small value of which indicates the stability of single reference CCSD calculations. In the table below, we compare our result for $\mathcal{E}_{\text {eff }}$ in $\mathrm{HgF}$ with previous calculations. Dmitriev et al. computed the value of $\mathcal{E}_{\text {eff }}$ in $\mathrm{HgF}$ using relativistic effective core potential calculations. They used the minimal atomic basis set for $\mathrm{F}$, and five relativistic valence orbitals $5 d_{3 / 2}, 5 d_{1 / 2}, 6 s_{1 / 2}, 6 p_{1 / 2}$, and $6 p_{3 / 2}$ for $\mathrm{Hg}$. Meyer et al. computed $\mathcal{E}_{\text {eff }}$ for $\mathrm{HgF}$ using 


\begin{tabular}{|l|c|}
\hline Work & $\mathcal{E}_{\text {eff }}(\mathrm{GV} / \mathrm{cm})$ \\
\hline Y Y Dmitriev et al. $[8]$ & 99.26 \\
Meyer et al. 24] & 68 \\
This work & 115.42 \\
\hline
\end{tabular}

TABLE II. Effective electric field, $\mathcal{E}_{\text {eff }}$, in the $\mathrm{HgF}$ molecule.

non-relativistic software to compare their method with results obtained by other methods.

We observe from the DF and CCSD values of $\mathcal{E}_{\text {eff }}$ that correlation effects contribute $\sim 10 \%$. A closer analysis of the eight terms that contribute to the correlation shows that there are cancellations between some of these terms. As an illustration of this point, in Table III we show the contributions of the individual terms to the expectation value in (7) for $\mathrm{HgF}$.

\begin{tabular}{|c|c|}
\hline Term & Contribution $(\mathrm{GV} / \mathrm{cm})$ \\
\hline $\mathrm{DF}$ & 104.25 \\
$H_{\mathrm{EDMM}}^{\text {eff }} T_{1}$ & 10.08 \\
$H_{\mathrm{EDM}}^{\text {eff }} T_{2}$ & 0 \\
$T_{1}^{\dagger} H_{\mathrm{EDM}}^{\text {eff }}$ & 10.08 \\
$T_{1}^{\dagger} H_{\mathrm{EDMM}}^{\text {eff }} T_{1}$ & -3.91 \\
$T_{1}^{\dagger} H_{\mathrm{EDM}}^{\text {eff }} T_{2}$ & 0.22 \\
$T_{2}^{\dagger} H_{\mathrm{EDM}}^{\text {eff }}$ & 0 \\
$T_{2}^{\dagger} H_{\mathrm{EDMM}}^{\text {eff }} T_{1}$ & 0.22 \\
$T_{2}^{\dagger} H_{\mathrm{EDM}}^{\text {eff }} T_{2}$ & -5.52 \\
\hline
\end{tabular}

TABLE III. Contributions from the individual terms to the effective electric field of $\mathrm{HgF}$.

We see that among the correlation terms, the $H_{\mathrm{EDM}}^{\mathrm{eff}} T_{1}$ and the $T_{1}^{\dagger} H_{\mathrm{EDM}}^{\mathrm{eff}}$ terms together contribute $20.16 \mathrm{GV} / \mathrm{cm}$. But the $T_{1}^{\dagger} H_{\mathrm{EDM}}^{\text {eff }} T_{1}$ and the $T_{2}^{\dagger} H_{\mathrm{EDM}}^{\text {eff }} T_{2}$ terms together contribute $-9.43 \mathrm{GV} / \mathrm{cm}$. The 9 correlation terms hence add up to $11.17 \mathrm{GV} / \mathrm{cm}$. Note that the $H_{\mathrm{EDM}}^{\mathrm{eff}} T_{2}$ term and the $T_{2}^{\dagger} H_{\mathrm{EDM}}^{\mathrm{eff}}$ are zero. This follows fom the application of slater-Condon rules 14 to an one-body operator, $H_{\mathrm{EDM}}$. The same reasoning applies, for example, also for the $H_{\mathrm{EDM}}^{\text {eff }} T_{1}^{2}$ term. The $H_{\mathrm{EDM}}^{\text {eff }} T_{1}$ term is the off-diagonal matrix element between the DF reference state, and a state with one electron excited by the electron-electron Coulomb repulsion.

The possible sources of error in our calculations stem from our choice of basis sets and not taking into account certain higher order correlation effects. From the difference in the effective electric field of $\mathrm{HgF}$ between the $\mathrm{TZ}$ and the DZ basis sets, we can estimate the error due to choice of basis to be around 1.5 percentage for all the mercury halides. A conservative estimate of the total error due to basis sets and omitting certain higher order correlation effects would be around 5 percentage.

The eEDM sensitivity of experiments is $\propto \mathcal{E}_{\text {eff }} \sqrt{N}$, where $N$ is the number of molecules whose spin precession is detected. In addition to their large effective electric fields, there is the particularly interesting possibility that $\mathrm{HgX}$ molecules can be produced in large quantities at ultracold temperatures, e.g. by photo-association of lasercooled $\mathrm{Hg}$ with magnetically trapped halogen atoms [25]. An intense and slow beam or fountain of $\mathrm{HgX}$ molecules could result in upto $\sim 1 \mathrm{~s}$ coherence time for electron spin precession. These molecules also offer a pathway for efficient detection: above their ground $X^{2} \Sigma$ state, they have a repulsive $A^{2} \Pi$ state which dissociates into $\mathrm{Hg}\left({ }^{1} S\right)$ and $\mathrm{X}\left({ }^{2} P\right)$ atoms. State-selective photo-dissociation of $\mathrm{HgX}$, coupled with laser-induced cycling fluorescence on the product $\mathrm{Hg}$ atom, can be used to detect spin precession in these molecules with unit efficiency. Molecules used in eEDM experiments must be fully polarized by lab electric fields in order to take full advantage of their effective electric fields. The quantity that sets the scale for the required lab electric field is $\mathcal{E}_{\mathrm{pol}}=2 B_{e} / D$, where $D$ is the molecular dipole moment and $B_{e}$ is the rotational constant of the molecule. Figure 2 shows the trend for $\mathrm{HgX}$ molecules, and picks out $\mathrm{HgBr}$ and $\mathrm{HgI}$ as attractive eEDM search candidates due to their combination of large $\mathcal{E}_{\text {eff }}$ and low $\mathcal{E}_{\text {pol }}$.

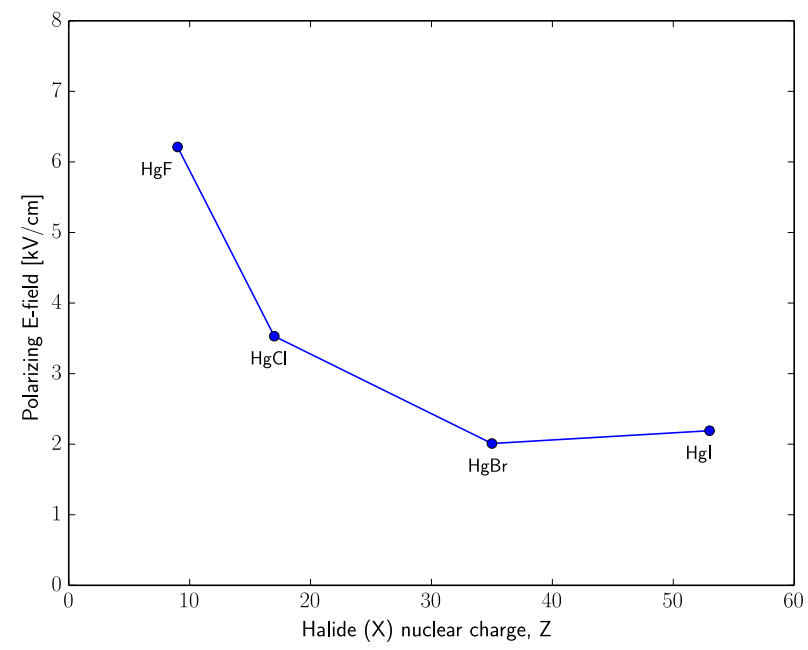

FIG. 2. Polarizing electric field, $\mathcal{E}_{\mathrm{pol}}=2 B_{e} / D$, for $\mathrm{HgX}$ molecules.

In summary, we have performed fully relativistic coupled cluster calculations of the effective electric fields in a family of molecules, the mercury monohalides. We find that these molecules have some of the largest effective electric fields known for polar diatomics, in addition to features that are favourable for experiments. This combination makes the mercury monohalides attractive candidates for the next generation of eEDM experiments.

\section{ACKNOWLEDGMENTS}

The computational results reported in this work were performed on the high performance computing facilities 
of IIA, Bangalore, on the Hydra and Kaspar clusters. We acknowledge Anish Parwage for his help with installing codes on the clusters. This research was supported by JST, CREST. M.A. thanks MEXT for financial support. The DiRef database was extremely useful in searching for literature [26].

[1] Michael Dine and Alexander Kusenko. Origin of the matter-antimatter asymmetry. Rev. Mod. Phys., 76(1):130, December 2003.

[2] Laurent Canetti, Marco Drewes, and Mikhail Shaposhnikov. Matter and antimatter in the universe. New J. Phys., 14(9):095012, September 2012.

[3] Norval Fortson, Patrick Sandars, and Stephen Barr. The search for a permanent electric dipole moment. Phys. Today, 56(6):33-39, 2003.

[4] Jonathan Engel, MJ Michael J. Ramsey-Musolf, U. van Kolck, and U Van Kolck. Electric dipole moments of nucleons, nuclei, and atoms: The standard model and beyond. Prog. Part. Nucl. Phys., 71:1-82, July 2013.

[5] The ACME Collaboration: J Baron, W C Campbell, David Demille, J M Doyle, G Gabrielse, Y V Gurevich, P W Hess, N R Hutzler, E Kirilov, I Kozyryev, B R O'Leary, C D Panda, M F Parsons, E S Petrik, B Spaun, A C Vutha, and A D West. Order of magnitude smaller limit on the electric dipole moment of the electron. Science, 343:269, January 2014.

[6] M R Tarbutt, B E Sauer, J J Hudson, and E A Hinds. Design for a fountain of $\mathrm{YbF}$ molecules to measure the electron's electric dipole moment. New J. Phys., 15(5):053034, May 2013.

[7] Timo Fleig and Malaya K. Nayak. Electron electric dipole moment and hyperfine interaction constants for ThO. J. Mol. Spectrosc., 300:16-21, June 2014.

[8] YY Dmitriev, YG Khait, and MG Kozlov. Calculation of the spin-rotational Hamiltonian including P-and P, T-odd weak interaction terms for $\mathrm{HgF}$ and $\mathrm{PbF}$ molecules. Phys. Lett. A, 1992.

[9] M. Abe, G. Gopakumar, M. Hada, B. P. Das, H. Tatewaki, and D. Mukherjee. Application of a Relativistic CoupledCluster Theory to the Effective Electric Field in YbF. May 2014.

[10] U. Kaldor E. Eliav and Y. Ishikawa. Int. J. Quantum Chem., (52):205, 1994.

[11] B. P. Das H. S. Nataraj, B. K. Sahoo and D. Mukherjee. Phys. Rev. Lett., (101):033002, 2008.
[12] V. Kvasnička, V. Laurinc, and S. Biskupič. Coupledcluster approach in electronic structure theory of molecules. Phys. Rep., 90(3):159-202, October 1982.

[13] The Coupled Cluster Method, 1997.

[14] J. Morrison I. Lindgren. Atomic Many-Body Theory, 2nd edition. Springer-Verlag, 1986.

[15] M.; Kawashima Y.; Nakajima T.; Nakano H.; Nakao. Y; Sekino H.; Paulovic J.; Tsuneda T.; Yanagisawa S.; Hirao K. UTChem 2004: Yanai, T.; Kamiya. 2004.

[16] M. Abe, H. Iikura, M. Kamiya, T. Nakajima, J. Paulovic, S. Yanagisawa, and Yanai. T. 2004.

[17] T. Nakajima M. Abe, T.Yanai and K. Hirao. Phys. Lett., (388):68, 2004.

[18] H. J. Aa. Jensen L. Visscher, T. Saue, K. G. Dyall U. Ekstrm E. Eliav T. Fleig A. S. P. Gomes T. U. Helgaker J. Hen-riksson M. Ilia Ch. R. Jacob S. Knecht P. Norman J. Olsen M. Pernpointner K. Ruud P. Saek R. Bast, S. Dubillard, and J. Sikkema. Dirac, a relativistic ab initio electronic structure pro- gram, release dirac08 (2008). 2008.

[19] K.L. Schuchardt, B.T. Didier, T. Elsetha-gen, L. Sun, V. Gurumoorthi, J. Chase, J. Li, and T.L. Windus. Basis set exchange: A community database for computational sciences. J. Chem. Inf. Model., (47(3)):1045-1052, March 2007.

[20] K.G. Dyall. Theor. Chem. Acc., 1998 (99:366), 2002 (108:365), 2006 (115:441).

[21] Dyall and Faegri. Introduction to Relativistic Quantum Chemistry, Dyall and Faegri, Oxford University Press. 2006.

[22] Stefan Knecht, Samuel Fux, Robert van Meer, Lucas Visscher, Markus Reiher, and Trond Saue. Mossbauer spectroscopy for heavy elements: a relativistic benchmark study of mercury. Theor. Chem. Acc., 129(3-5):631-650, 2011.

[23] Nai-ho Cheung and TA Cool. Franck-Condon factors and r-centroids for the B 2 Sigma - X 2 Sigma systems of $\mathrm{HgCl}, \mathrm{HgBr}$ and $\mathrm{HgI}$. J. Quant. Spectrosc. Radiat. Transf., 21(10), 1979.

[24] Michael P. Deskevich Edmund R. Meyer, John L. Bohn. Candidate molecular ions for an electron electric dipole moment experiment. Physical Review A, (73):062108, 2006.

[25] C. J. Rennick, J. Lam, W. G. Doherty, and T. P. Softley. Magnetic Trapping of Cold Bromine Atoms. Phys. Rev. Lett., 112(2):023002, January 2014.

[26] P.F. Bernath and S. McLeod. Diref, a database of references associated with the spectra of diatomic molecules. J. Mol. Spectrosc., 207:287, 2001. 